\title{
Estudo dos fatores impregnação e secagem nas características de glóbulos utilizados em homeopatia
}

\author{
Eliana E. Diehl*,1, Diva Sonaglio², Nayla Ferreira Lima², Sinara Backes ${ }^{1}$ \\ ${ }^{3}$ Laboratório de Homeopatia, Departamento de Ciências Farmacêuticas, Centro de Ciências da Saúde, Universidade \\ Federal de Santa Catarina, ${ }^{2}$ Laboratório de Farmacotécnica, Departamento de Ciências Farmacêuticas, Centro de \\ Ciências da Saúde, Universidade Federal de Santa Catarina
}

${ }^{*}$ Correspondência:

E. E. Diehl

Laboratório de Homeopatia

Departamento de Ciências

Farmacêuticas

Centro de Ciências da Saúde

Universidade Federal de Santa

Catarina

Campus Universitário

88040-900 - Florianópolis, SC, Brasil E-mail: elianadieh|@hotmail.com
Glóbulos são uma das formas farmacêuticas mais dispensadas em farmácias homeopáticas no Brasil. Este trabalho teve como objetivo comparar técnicas de impregnação especificadas na Farmacopéia Homeopática Brasileira $2^{a}$ Edição e Manual de Normas Técnicas para Farmácia Homeopática $3^{a}$ Edição e na prática em farmácias homeopáticas do município de Florianópolis, Santa Catarina, Brasil. As variáveis de formulação percentual de insumo ativo (3 e 10\%) e tipo de impregnação (simples e tríplice) e a variável de processo temperatura de secagem $\left(20\right.$ e $\left.50^{\circ} \mathrm{C}\right)$ foram analisadas através de planejamento fatorial $2^{3}$. As respostas estudadas foram o peso antes e após impregnação e o tempo de desagregação. Os resultados mostraram maior diferença de peso com impregnação a $10 \%$ e secagem a $50^{\circ} \mathrm{C}$, independente do tipo de impregnação. O tempo de desagregação não foi influenciado pelas variáveis em estudo $(p<0,05)$. Verificou-se melhor homogeneidade para a formulação com impregnação tríplice a $10 \%$.

\section{INTRODUÇÃO}

A homeopatia consiste em ministrar ao paciente medicamentos diluídos e dinamizados, visando estimular a reação orgânica e evitar a agravação dos sintomas (Fontes, 2005). A palavra Homeopatia tem origem do grego homoios "semelhante" e pathos "doença", respeitando o princípio hipocrático da semelhança similia similibus curantur. Para Christian Friedrich Samuel Hahnemann (1755-1843), médico alemão fundador da homeopatia, os medicamentos homeopáticos têm a capacidade de curar porque "seus sintomas são semelhantes aos da doença e superiores a ela em força” (Hahnemann, 2001).

Hahnemann catalogou os efeitos de diversas substâncias no organismo humano sadio e passou a prescrevê-las para indivíduos doentes, obtendo resultados positivos. Ainda com a finalidade de diminuir os efeitos tóxicos dos medicamentos e suas interações, adotou as doses infinitesimais (diluições seguidas de dinamização) e o medicamento único. A dinamização é um procedimento técnico mecânico no qual as diluições prévias em meio adequado são submetidas a um processo vigoroso de agitação ou atrito, desenvolvendo assim a força medicamentosa latente das substâncias (Hahnemann, 2001). 
Em 1805, Hahnemann publicou a primeira matéria médica homeopática com 27 substâncias testadas e, em 1810, a primeira edição de seu livro básico Organon da arte de curar, onde se encontram a doutrina homeopática e seus ensinamentos. Nas várias edições (seis no total) do Organon, apresentam-se técnicas de preparação do medicamento homeopático. Essas técnicas foram apropriadas em diversas farmacopéias no mundo inteiro, como a Pharmacopoea homeopathica polyglotta de Wilmar Schwabe, publicada pela primeira vez em 1894, que foi referência até recentemente em muitos países, incluindo o Brasil.

No Brasil, a homeopatia foi introduzida em 1840 pelo médico francês Benoit Jules Mure, difundindo-se por todo o país. Em 1980, foi reconhecida como especialidade médica pelo Conselho Federal de Medicina (Luz, 1996). Em relação às preparações homeopáticas, até 1977 eram feitas de acordo com farmacopéias e códigos de outros países. Nesse mesmo ano, foi publicada a primeira edição da Farmacopéia Homeopática Brasileira. Nos anos seguintes, não houve uma padronização sistemática nos métodos e técnicas de preparação dos medicamentos homeopáticos, permanecendo a forte influência de outros países, como França e Alemanha.

Em 1992, a Associação Brasileira de Farmacêuticos Homeopatas (ABFH) lançou a primeira edição do Manual de Normas Técnicas para Farmácia Homeopática, que tinha como um dos objetivos principais "apresentar aos profissionais um conjunto de informações a respeito dos procedimentos gerais envolvendo a origem, preparação, conservação, dispensação e outras características dos medicamentos homeopáticos" (ABFH, 1992). A partir de então, cada vez mais se estimulou a padronização no preparo dos insumos e medicamentos homeopáticos e a segunda edição da Farmacopéia Homeopática Brasileira (Farm. Hom. Bras., 1997) procurou atingir essa meta.

De acordo com a Farm. Hom. Bras. (1997), o "medicamento homeopático é toda apresentação farmacêutica destinada a ser ministrada segundo o princípio da similitude, com finalidade preventiva e terapêutica, obtida pelo método de diluições seguidas de sucussões e/ou triturações sucessivas". Os medicamentos homeopáticos para uso interno encontram-se nas formas farmacêuticas líquidas (gotas e dose única líquida) e sólidas (comprimidos, glóbulos, pós, tabletes e dose única sólida).

Os glóbulos são obtidos industrialmente a partir de sacarose ou de mistura de sacarose e lactose, com pesos médios de 30, 50 e $70 \mathrm{mg}$, que correspondem aos números 3,5 e 7, respectivamente. São esferas homogêneas e regulares, de cor branca, inodoros e de sabor adocicado (Farm. Hom. Bras., 1997). As farmácias homeopáticas adquirem os glóbulos na forma inerte e a impregnação pelo insumo ativo deve ser feita seguindo especificações descritas na Farmacopéia Homeopática Brasileira (1997) ou na terceira edição do Manual de Normas Técnicas (ABFH, 2003).

O processo de impregnação é descrito de maneira diferente nessas duas referências. De acordo com a Farmacopéia Homeopática Brasileira (1997), os glóbulos são impregnados na forma da tríplice impregnação com $10 \%(\mathrm{~V} / \mathrm{p})$ do insumo ativo e a secagem é feita em estufa até $50^{\circ} \mathrm{C}$. Segundo o Manual de Normas Técnicas (2003), a impregnação pode ser realizada na forma simples ou tríplice, percentual de insumo ativo de $2 \%(\mathrm{~V} / \mathrm{p})$ a $5 \%(\mathrm{~V} /$ p) e secagem em temperatura ambiente ou inferior a $40^{\circ} \mathrm{C}$.

Esse trabalho tem como objetivo analisar a técnica de impregnação dos glóbulos, uma das formas farmacêuticas mais dispensadas na farmácia homeopática no Brasil, comparando as técnicas especificadas na Farmacopéia Homeopática Brasileira (1997) e no Manual de Normas Técnicas (2003). Os resultados têm potencial contribuição à prática cotidiana das farmácias homeopáticas, já que poderão auxiliar na definição das condições mais adequadas para a impregnação dessa forma farmacêutica.

\section{MATERIAL E MÉTODOS}

\section{Material}

Para os ensaios utilizaram-se glóbulos inertes $\mathrm{n}^{0} 7$ (Laboratório Scharaibmann Ltda.), álcool de cereais $96^{\circ} \mathrm{GL}$ (Labsynth Produtos para Laboratórios Ltda.), solução hidroalcoólica $\left(70^{\circ} \mathrm{GL}\right)$ de violeta de genciana $1 \%(\mathrm{p} /$ V), balança analítica (Gibertini E42S-B), estufa (Fanem 315 SE), aparelho de desintegração (Nova Ética 301-AC).

\section{Métodos}

\section{Preparação dos glóbulos}

Pesaram-se, em balança analítica, 24 amostras de aproximadamente $10 \mathrm{~g}$ de glóbulos inertes, em frasco de vidro âmbar com capacidade de $60 \mathrm{~mL}$, onde foram realizadas as impregnações com a solução de violeta de genciana $1 \%(\mathrm{p} / \mathrm{V})$.

Realizaram-se os experimentos alterando variáveis (A, B e C) de formulação (percentual de impregnação e tipo de impregnação) e de processo (temperatura de secagem), em dois níveis, inferior (-) e superior $(+)$, conforme representado na Tabela I.

Para os experimentos em que a impregnação foi a 3\% simples (S), utilizou-se pipeta automática com volume ajustado para $0,30 \mathrm{~mL}$; para a impregnação a $3 \%$ tríplice (T), a pipeta automática foi ajustada com 1/3 do volume, ou seja, $0,10 \mathrm{~mL}$ adicionados três vezes, sendo que, após 
TABELA I - Fatores e níveis do planejamento fatorial $2^{3}$

\begin{tabular}{lcc}
\hline Variáveis & \multicolumn{2}{c}{ Níveis } \\
& $\begin{array}{c}\text { Inferior } \\
(-)\end{array}$ & $\begin{array}{c}\text { Superior } \\
(+)\end{array}$ \\
\hline A (Percentual de impregnação) & $3 \%$ & $10 \%$ \\
B (Temperatura de secagem) & $20^{\circ} \mathrm{C}$ & $50^{\circ} \mathrm{C}$ \\
C (Tipo de impregnação) & Simples & Tríplice \\
& $(\mathrm{S})$ & $(\mathrm{T})$ \\
\hline
\end{tabular}

cada adição, agitou-se o frasco durante um minuto.

Nos glóbulos impregnados com solução a $10 \%$, adicionou-se o volume de $1,0 \mathrm{~mL}$ na impregnação simples e duas vezes $0,35 \mathrm{~mL}$ e mais uma vez $0,30 \mathrm{~mL}$ na impregnação tríplice, agitando-se após cada adição. A secagem das amostras foi realizada à temperatura ambiente de $20^{\circ} \mathrm{C}$ $\left( \pm 2^{\circ} \mathrm{C}\right)$, mantida através de equipamento de ar condicionado, e em estufa a $50^{\circ} \mathrm{C}\left( \pm 2^{\circ} \mathrm{C}\right)$.

\section{Caracterização dos glóbulos}

Após impregnação e secagem das amostras realizouse nova pesagem. A "diferença de peso" $\left(\mathrm{P}_{\mathrm{f}}-\mathrm{P}_{\mathrm{i}}\right)$, observada antes (Pi) e após a impregnação e secagem (Pf) dos glóbulos, foi analisada estatisticamente (Resposta 1).

O ensaio de desintegração, aqui designado desagregação de acordo com a Farmacopéia Homeopática Brasileira (1997), foi realizado em conformidade com a técnica para comprimidos e cápsulas da Farmacopéia Brasileira (1988), que consiste em um sistema de cestas contendo 6 tubos cada, imersos em água. Padronizou-se que cada cesta representaria uma amostra (glóbulos não impregnados e impregnados previamente a $3 \%$ e a $10 \%$, S e T), sendo adicionado um glóbulo por tubo. Submeteram-se os glóbulos a movimentos verticais, com velocidade e freqüência constantes, e determinou-se o "tempo de desagregação", o qual também foi analisado estatisticamente (Resposta 2).

\section{Planejamento estatístico e análise}

As três variáveis (ou fatores) a dois níveis (Tabela I) analisadas neste delineamento foram escolhidas com base na literatura (Farm. Hom. Bras., 1997; ABFH, 2003) e na prática vigente em farmácias homeopáticas do município de Florianópolis, estado de Santa Catarina (SC). Realizaram-se os experimentos em triplicata, seguindo o planejamento fatorial $2^{3}$, de forma aleatória, totalizando 24 ensaios (Tabela II). Conforme Tabela I, os fatores avaliados foram: $\mathrm{A}=$ percentual de insumo ativo $3 \% \mathrm{~V} / \mathrm{p}$ (prática em farmácia/SC; ABFH, 2003) e 10\% V/p (Farm. Hom. Bras., 1997); $\mathrm{B}=$ temperatura ambiente $20^{\circ} \mathrm{C}$ (conforme prática em farmácia/SC) e $50^{\circ} \mathrm{C}$ (preconizado pela Farm. Hom.
Bras., 1997); $\mathrm{C}=$ tipo de impregnação simples (ABFH, 2003) ou tríplice (Farm. Hom. Bras., 1997; ABFH, 2003). Analisaram-se as respostas $(\mathrm{Y})$ "diferença de peso" $\left(\mathrm{P}_{\mathrm{f}}-\mathrm{P}_{\mathrm{i}}\right)$ (em mg) e "tempo de desagregação" (em segundos), utilizando software Design Expert, versão 6.0.6 (StatEase, Minneapolis, MN). A equação de regressão resultante do planejamento fatorial é do tipo:

$\mathrm{Y}=\mathrm{b}_{0}+\mathrm{b}_{1} \mathrm{~A}+\mathrm{b}_{2} \mathrm{~B}+\mathrm{b}_{3} \mathrm{C}+\mathrm{b}_{12} \mathrm{AB}+\mathrm{b}_{13} \mathrm{AC}+\mathrm{b}_{23} \mathrm{BC}+$ $\mathrm{b}_{123} \mathrm{ABC}$

onde $\mathrm{Y}$ é a resposta do modelo, $\mathrm{b}_{0}$ é a média dos valores, $\mathrm{b}_{1}$, $\mathrm{b}_{2}, \mathrm{~b}_{3}$ os coeficientes dos efeitos principais (A, B, C), $\mathrm{b}_{12}$, $\mathrm{b}_{13}, \mathrm{~b}_{23}$ os coeficientes das interações entre dois fatores $(\mathrm{AB}$, $\mathrm{AC}, \mathrm{BC})$ e $\mathrm{b}_{123}$ o coeficiente da interação entre os 3 fatores (ABC). A análise da variância (ANOVA) foi realizada para cada resposta empregando-se uma probabilidade de erro de $p=0,05$ ou $p=0,10$. Os termos que aparecem na equação foram determinados por análise hierárquica. $\mathrm{O}$ delineamento fatorial completo $2^{3}$, com três repetições $(n=3)$, fornece suficientes graus de liberdade para discriminação estatística entre os fatores principais ( $\mathrm{A}, \mathrm{B}$ e C), as interações binárias $(\mathrm{AB}, \mathrm{AC}, \mathrm{e} \mathrm{BC})$ e ternária $(\mathrm{ABC})$ (Montgomery, 2001; Howard, Neau, Sack, 2006).

TABELA II - Delineamento segundo planejamento fatorial $2^{3}$

\begin{tabular}{lccc}
\hline Experimento & \multicolumn{3}{c}{ Fatores } \\
& $\begin{array}{c}\text { A } \\
\text { Insumo } \\
\text { Ativo }(\%)\end{array}$ & $\begin{array}{c}\text { Temperatura } \\
\left({ }^{\circ} \mathrm{C}\right)\end{array}$ & $\begin{array}{c}\mathrm{C} \\
\text { Tipo de } \\
\text { impregnação }\end{array}$ \\
\hline 1 & - & - & - \\
2 & + & - & - \\
3 & - & + & - \\
4 & + & + & - \\
5 & - & - & + \\
6 & + & - & + \\
7 & - & + & + \\
8 & + & + & + \\
\hline
\end{tabular}

\section{RESULTADOS E DISCUSSÃO}

Planejamentos estatísticos denominados delineamentos fatoriais são usados primeiramente para realizar um screening dos fatores significantes, mas também podem ser utilizados para modelar e refinar uma análise (ou processo). Neste trabalho foi utilizado um delineamento fatorial $2^{3}$ como instrumento para comparar as diferentes técnicas de impregnação de insumo ativo em glóbulos inertes. $\mathrm{O}$ 
Manual de Normas Técnicas (ABFH, 2003) e outros trabalhos (Sartori et al., 2003; Araújo et al., 2004) indicam o corante violeta de genciana como o mais adequado para uma rápida, regular e homogênea impregnação quando se está validando as técnicas de impregnação de glóbulos homeopáticos. Informações obtidas a partir da literatura (Farm. Hom. Bras., 1997; ABFH, 2003) e da prática em farmácias de manipulação no município de Florianópolis, $\mathrm{SC}$, identificaram diferenças nas técnicas, especialmente relativas às variáveis de formulação (percentual de impregnação e tipo de impregnação) e de processo (temperatura de secagem). Estas variáveis foram então escolhidas para análise e avaliação de sua influência sobre o produto final.

Os fatores percentual de impregnação (A) e temperatura de secagem (B) foram caracterizados como numéricos (ou quantificáveis) e o fator tipo de impregnação (C) como categórico, não podendo, portanto ser quantificado. As respostas (ou resultados) utilizadas para caracterizar os diferentes fatores envolvidos foram a "diferença de peso" antes e após a impregnação $\left(\mathrm{P}_{\mathrm{f}}-\mathrm{P}_{\mathrm{i}}\right)$ e o "tempo de desagre- gação". O modelo de delineamento utilizado foi a interação entre os três fatores $(3 \mathrm{FI})$.

Os valores das respostas "diferença de peso" $\left(\mathrm{Y}_{1}\right)$ e "tempo de desagregação" $\left(\mathrm{Y}_{2}\right)$ obtidas a partir dos glóbulos impregnados nas diferentes condições experimentais são apresentados na Tabela III.

A análise da variância (ANOVA) do modelo fatorial para a resposta "diferença de peso" $\left(\mathrm{P}_{\mathrm{f}}-\mathrm{P}_{\mathrm{i}}\right)$ está representada na Tabela IV. Os valores obtidos variaram de $-0,0021$ a $0,6766 \mathrm{mg}$ (Tabela III). AANOVA, para o modelo fatorial selecionado, apresenta um valor de $\mathrm{F}=256,12$, indicando que o modelo é significante $(p<0,0001)$. Valores de Prob $>F$ menores que $0,05(p=0,05)$ ou $0,10(p=0,10)$ indicam que os termos são significantes. Neste modelo, verifica-se que os fatores percentual de impregnação (A), temperatura de secagem $(\mathrm{B})$ e as interações $\mathrm{AB}, \mathrm{AC}$ e $\mathrm{ABC}$ são significantes quando se considera um $p=0,05$. Para um $p=$ 0,10 , o termo C (tipo de impregnação) também torna-se significante. Considerando o alto valor do coeficiente de correlação $\left(\mathrm{R}^{2}=0,9923\right)$, pode-se esperar uma alta predição,

TABELA III - Planejamento com os respectivos fatores e respostas

\begin{tabular}{lccccc}
\hline $\begin{array}{l}\mathrm{N}^{\circ} \text { do } \\
\text { experimento }\end{array}$ & $\begin{array}{c}\text { A (Insumo } \\
\text { ativo }(\%)\end{array}$ & $\begin{array}{c}\mathrm{B}(\text { Temperatura de } \\
\text { secagem })\left({ }^{\circ} \mathrm{C}\right)\end{array}$ & $\begin{array}{c}\mathrm{C}(\text { Tipo de } \\
\text { impregnação })\end{array}$ & $\begin{array}{c}\mathrm{Y}_{1}\left(\mathrm{P}_{\mathrm{f}}-\mathrm{P}_{\mathrm{i}}\right) \\
(\mathrm{mg})\end{array}$ & $\begin{array}{c}\mathrm{Y}_{2}(\text { Tempo de } \\
\text { desagregação })(\mathrm{s})\end{array}$ \\
\hline 1 & 3 & 20 & $\mathrm{~S}$ & 0,2196 & 64 \\
2 & 3 & 20 & $\mathrm{~S}$ & 0,2148 & 118 \\
3 & 3 & 20 & $\mathrm{~S}$ & 0,2140 & 92 \\
4 & 10 & 20 & $\mathrm{~S}$ & $-0,0194$ & 86 \\
5 & 10 & 20 & $\mathrm{~S}$ & 0,0020 & 94 \\
6 & 10 & 20 & $\mathrm{~S}$ & 0,0116 & 114 \\
7 & 3 & 50 & $\mathrm{~S}$ & 0,2178 & 110 \\
8 & 3 & $\mathrm{~S}$ & 0,2207 & 84 \\
9 & 3 & $\mathrm{~S}$ & 0,2267 & 117 \\
10 & 10 & $\mathrm{~S}$ & 0,5147 & 106 \\
11 & 10 & 50 & $\mathrm{~S}$ & 0,4302 & 112 \\
12 & 10 & 50 & $\mathrm{~S}$ & 0,4377 & 100 \\
13 & 3 & 50 & $\mathrm{~T}$ & 0,2879 & 106 \\
14 & 3 & 20 & $\mathrm{~T}$ & 0,2310 & 73 \\
15 & 3 & $\mathrm{~T}$ & 0,2342 & 90 \\
16 & 10 & 20 & $\mathrm{~T}$ & 0,0121 & 83 \\
17 & 10 & $\mathrm{~T}$ & $-0,0255$ & 117 \\
18 & 10 & $\mathrm{~T}$ & $-0,0021$ & 86 \\
19 & 20 & $\mathrm{~T}$ & 0,0511 & 104 \\
20 & 3 & $\mathrm{~T}$ & 0,0606 & 104 \\
21 & 3 & $\mathrm{~T}$ & 0,1001 & 118 \\
22 & 3 & 50 & $\mathrm{~T}$ & 0,6690 & 110 \\
23 & 10 & 50 & $\mathrm{~T}$ & 0,6132 & 120 \\
24 & 10 & 50 & & 0,6766 & \\
\hline
\end{tabular}


o que é confirmado pelo também alto valor de $R^{2}$ preditivo $(0,9772)$ fornecido pelo modelo fatorial.

TABELA IV - ANOVA para o modelo fatorial selecionado com a resposta "diferença de peso"

\begin{tabular}{lcc}
\hline Fonte & Valor de F & $\begin{array}{c}p \\
\text { Prob }>\mathrm{F}\end{array}$ \\
\hline Modelo & 256,12 & $<0,0001$ \\
$\mathrm{~A}$ & 73,55 & $<0,0001$ \\
$\mathrm{~B}$ & 546,11 & $<0,0001$ \\
$\mathrm{C}$ & 3,22 & 0,0945 \\
$\mathrm{AB}$ & 1024,40 & $<0,0001$ \\
$\mathrm{AC}$ & 56,78 & $<0,0001$ \\
$\mathrm{BC}$ & 0,05 & 0,8223 \\
$\mathrm{ABC}$ & 88,73 & $<0,0001$ \\
Desvio padrão & 0,02 & \\
Média & 0,23 & \\
C.V. & 10,63 & \\
$R^{2}$ & 0,9923 & \\
$R^{2}$ Preditivo & 0,9772 & \\
\hline
\end{tabular}

A análise da variância (ANOVA) do modelo fatorial para a resposta "tempo de desagregação" está representada na Tabela V. Os valores obtidos variaram de 64 a 120 segundos (Tabela III). A ANOVA, para o modelo fatorial selecionado, apresenta um valor de $\mathrm{F}=3,17$, indicando que o modelo só é significante para uma probabilidade de erro de $p=0,10(p<0,09)$. Neste modelo, verifica-se que apenas o fator $\mathrm{B}$ (temperatura de secagem) teria uma pequena influência na resposta $(p<0,09)$.

TABELA V - ANOVA para o modelo fatorial selecionado com a resposta "tempo de desagregação"

\begin{tabular}{lcc}
\hline Fonte & Valor de F & $\begin{array}{c}p \\
\text { Prob }>\text { F }\end{array}$ \\
\hline Modelo & 3,17 & 0,0904 \\
B & 3,17 & 0,0904 \\
Desvio padrão & 0,14 & \\
Média & 0,09 & \\
C.V. & $-0,24$ & \\
\hline
\end{tabular}

\section{Resposta 1 (Y): Diferença de peso}

A equação final do modelo para os fatores analisados relacionada à resposta "diferença de peso" é:
$\mathrm{Y}_{1}\left(\mathrm{P}_{\mathrm{f}}-\mathrm{P}_{\mathrm{i}}\right)=0,2333+0,0434 \mathrm{~A}+0,1183 \mathrm{~B}+0,1620 \mathrm{AB}$ $+0,0381 \mathrm{AC}+0,0477 \mathrm{ABC}$

Observa-se que o fator tipo de impregnação (C) não está representado, pois não é significante (Tabela IV). Entretanto, como está envolvido nas interações, deverá ser analisado juntamente com os demais fatores. Analisandose as Figuras 1 e 2, verifica-se que tanto para as impregnações do tipo simples $(\mathrm{S})$ quanto tríplice $(\mathrm{T})$, analisadas separadamente, ocorre uma maior "diferença de peso" $\left(\mathrm{P}_{\mathrm{f}}\right.$ - $\mathrm{P}_{\mathrm{i}}$ ) quando os dois fatores estão no seu nível máximo, ou seja, impregnação a $10 \%$ e secagem a $50^{\circ} \mathrm{C}$.

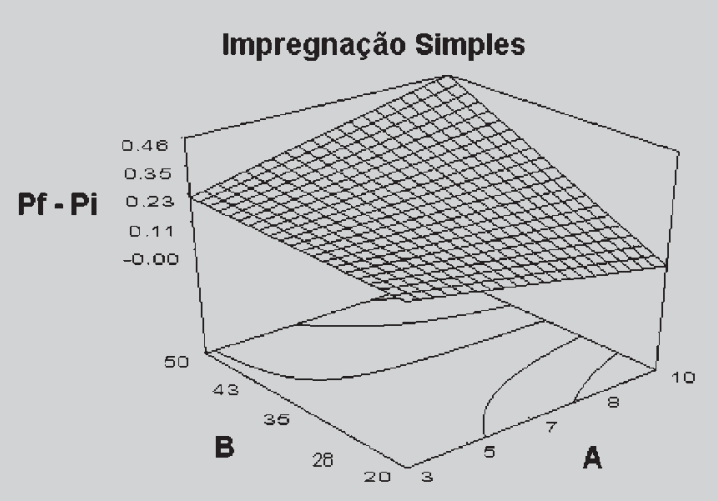

FIGURA 1 - Modelo gráfico da interação entre os fatores percentual de impregnação (A) e temperatura de secagem (B) dos glóbulos com impregnação simples (S) em função da "diferença de peso" $\left(\mathrm{P}_{\mathrm{f}}-\mathrm{P}_{\mathrm{i}}\right)$.

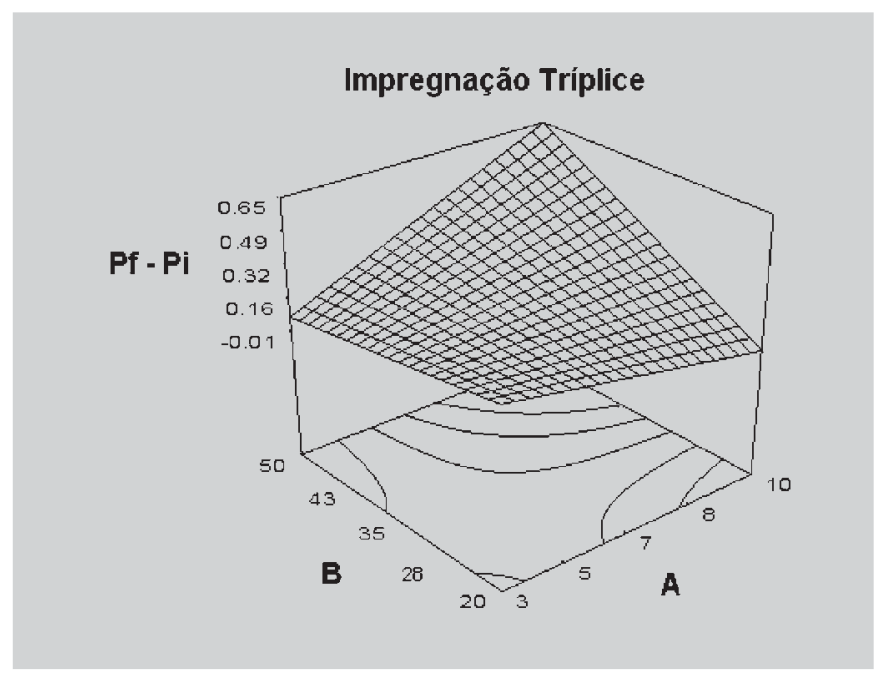

FIGURA 2 - Modelo gráfico da interação entre os fatores percentual de impregnação (A) e temperatura de secagem (B) dos glóbulos com impregnação tríplice (T) em função da "diferença de peso" $\left(\mathrm{P}_{\mathrm{f}}-\mathrm{P}_{\mathrm{i}}\right)$. 
Este resultado era esperado com relação ao aumento do percentual de impregnação de $3 \%$ para $10 \%$. Entretanto, aumentando-se a temperatura de secagem de $20^{\circ} \mathrm{C}$ para $50^{\circ} \mathrm{C}$, podia-se esperar uma diminuição da "diferença de peso". Este comportamento pode ser explicado pelas perdas ocorridas durante o processo de impregnação com o fator A no nível máximo (10\%) e o fator B no nível mínimo $\left(20^{\circ} \mathrm{C}\right)$. Com maior quantidade de líquido e o prolongamento do tempo de secagem, os glóbulos aderiram-se às paredes dos frascos, sofrendo perda de sua massa no momento da pesagem. Essa massa aderida às paredes é proporcional à variação observada na diferença de peso e, mesmo sendo da ordem de décimos ou centésimos de miligrama, é estatisticamente significativa. Quando os três fatores são analisados concomitantemente (Figura 3), observa-se que o maior aumento de peso $(0,6766 \mathrm{mg})$ ocorre quando os três fatores estão no seu nível máximo.

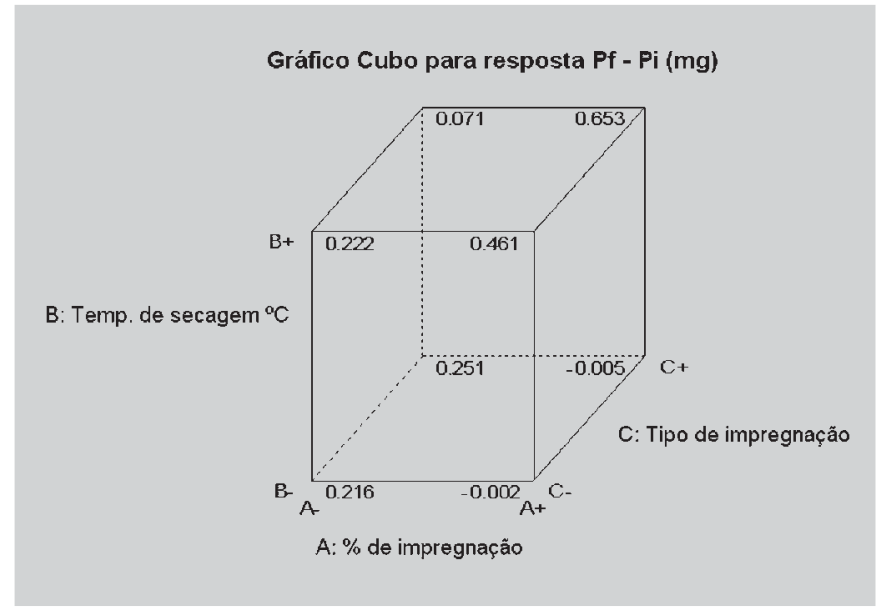

FIGURA 3 - Modelo gráfico da interação entre os três fatores para a resposta "diferença de peso" $\left(\mathrm{P}_{\mathrm{f}}-\mathrm{P}_{\mathrm{i}}\right)$.

\section{Resposta 2 (Y ): Tempo de desagregação}

A equação final do modelo para os fatores analisados com relação à resposta "tempo de desagregação" é:

$\mathrm{Y}_{2}=100,67+5,25 \mathrm{~B}$

Para esta resposta, apenas o efeito da temperatura de secagem tem pequena influência na resposta $(p=0,10)$, cujo aumento no seu nível significa um aumento no "tempo de desagregação". Se for considerada uma probabilidade de $5 \%$ de significância $(p=0,05)$, o fator temperatura não apresentaria influência na resposta. Esse resultado também foi observado por Sartori et al. (2003), que avaliaram o aspecto e a desagregação de glóbulos utilizados em homeopatia. A rápida desagregação está muito prova- velmente relacionada com a composição (sacarose ou mistura de sacarose e lactose) e com o método de fabricação dos glóbulos. Estes são produzidos a partir de cristais do açúcar, os quais são cobertos por camadas sucessivas e concêntricas do açúcar, semelhante ao processo de drageamento (Araújo et al., 2004).

Conforme preconiza o Manual de Normas Técnicas (ABFH, 2003), a desagregação deve ocorrer em, no máximo, dez minutos. Portanto, os resultados encontrados neste trabalho estão de acordo com o especificado nesse Manual. Entretanto, a Farm. Hom. Bras. (1997) preconiza que o tempo deve ser "da ordem de dez minutos", deixando dúvidas quanto à interpretação. Observando-se a Figura 4 constata-se o aumento no "tempo de desagregação" quando se eleva a temperatura de secagem (fator B) de $20^{\circ} \mathrm{C}$ para $50^{\circ} \mathrm{C}$. Este fato não acontece com o aumento do percentual de impregnação (fator A), cuja resposta permanece estável mesmo quando o percentual de insumo ativo é três vezes superior.

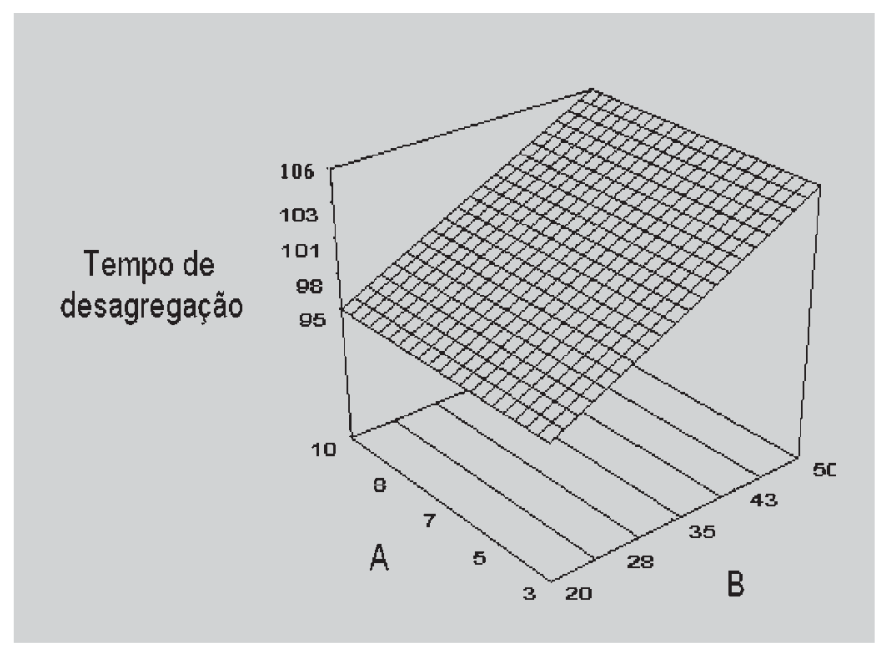

FIGURA 4 - Representação gráfica dos fatores percentual de impregnação (A) e temperatura de secagem (B) em função do "tempo de desagregação" dos glóbulos impregnados.

Quanto aos aspectos organolépticos, a formulação com impregnação tríplice (T) a 10\% apresentou a melhor homogeneidade. Porém, para evitar a agregação dos glóbulos e conseqüente perda de massa, a secagem deveria ser realizada em estufa a $50^{\circ} \mathrm{C}$. Nestas condições, observou-se um acréscimo de 2,7 vezes no peso (de 0,251 a $0,6766 \mathrm{mg}$ ). Portanto, utilizando-se todos os fatores analisados neste estudo nos seus níveis superiores, obtém-se um valor máximo no aumento de peso. Com relação à desagregação, não se observou influência dos fatores analisados $(p=0,05)$ no campo de experimentação estudado. Os 
baixos valores e a pequena variação observados na resposta "tempo de desagregação" podem ser atribuídos à composição dos glóbulos e à semelhança nas características destes, tais como igual tamanho, um único lote e um único fornecedor.

\section{CONCLUSÃO}

Os resultados obtidos neste trabalho demonstram a influência das principais variáveis de formulação. A combinação correta das variáveis percentual de impregnação, tipo de impregnação e temperatura de secagem são fatores essenciais para uma impregnação uniforme do insumo ativo nos glóbulos inertes. Da mesma forma, a alteração no percentual de impregnação do insumo ativo deve considerar os três fatores concomitantemente.

Procedimentos observados na prática em farmácias, com relação à resposta "diferença de peso", corroboram os resultados aqui apresentados. Uma impregnação do corante a $3 \%(\mathrm{~V} / \mathrm{p})$ poderia ser realizada por impregnação tríplice e secagem em temperatura de $20^{\circ} \mathrm{C}$, porém esse percentual apresenta o risco de comprometer a homogeneidade de conteúdo. Os resultados desse trabalho sugerem que a técnica mais adequada a ser utilizada é a impregnação tríplice a $10 \%$, com secagem a uma temperatura de $50^{\circ} \mathrm{C}$, por apresentar melhor uniformidade de coloração, ou seja, melhor uniformidade de conteúdo, além de evitar agregação dos glóbulos e perda de massa. Estas condições, entretanto, contrastam com aquelas de uso freqüente nas farmácias de manipulação no município de Florianópolis.

A impregnação dos glóbulos com corante reflete o comportamento de impregnação quando um insumo ativo homeopático é incorporado. De acordo com os princípios da homeopatia, os insumos ativos que impregnam os glóbulos estão na forma de soluções extremamente diluídas e dinamizadas, e não se esperaria, portanto, uma grande variação na massa dos mesmos. Assim, qualquer variação, fundamentalmente relacionada à homogeneidade de conteúdo dos glóbulos, interfere na qualidade do produto final, e deveria ser evitada através de otimização e padronização dos procedimentos tecnológicos utilizados.

Embora não existam evidências de que variações nas técnicas de impregnação de glóbulos possam comprometer a eficácia e efetividade clínicas dos medicamentos homeopáticos, a questão da padronização das técnicas permanece um desafio. Desde Hahnemann, que em nenhuma de suas publicações indicou uma proporção exata entre o volume do insumo ativo e o peso de glóbulos, até as várias farmacopéias homeopáticas e manuais existentes, não há consenso sobre esse tema. No Brasil, a partir de alguns trabalhos (Rocha et al., 2000; Gutierrez, 2001; Fontes et al., 2002; Pozetti, Silva, Pizzolitto, 2002; Sartori et al., 2003; Araújo et al., 2004; Pinheiro, 2006) que registram a validação da técnica de impregnação de glóbulos, observase a dificuldade de obtenção de conclusões convergentes quanto ao percentual de impregnação, tipo de impregnação e temperatura de secagem. Neste sentido, recomendam-se estudos mais aprofundados que poderão subsidiar uma orientação e padronização da literatura oficial.

\section{AGRADECIMENTOS}

Ao Farmacêutico Ângelo Fiamoncini, pela doação dos glóbulos inertes e álcool de cereais para a realização dos ensaios. Aos responsáveis pelo Laboratório de Controle de Qualidade do Departamento de Ciências Farmacêuticas da Universidade Federal de Santa Catarina, pelo apoio na parte experimental do trabalho.

\section{ABSTRACT}

\section{Study of impregnation and drying factors in the characteristics of globules used in homeopathy}

Globules are one of the most dosage forms dispensed in homeopathic pharmacies in Brazil. This work aimed at comparing different impregnation techniques specified in the Brazilian Homeopathic Pharmacopoeia 2 Edition and Manual of Technical Norms for Homeopathic Pharmacy 3 Edition and the practical in homeopathic pharmacies in the city of Florianópolis, state of Santa Catarina, Brazil. The formulations variables percent active raw material (3 and 10\%), impregnation type (simple and triple) and the process variable drying temperature $\left(20\right.$ and $\left.50^{\circ} \mathrm{C}\right)$ were analyzed through a factorial design $2^{3}$. The studied answers were the weight before and after the impregnation and time of disaggregation. The results show a larger weight difference with the 10\% impregnation and the drying of $50^{\circ} \mathrm{C}$, regardless of the impregnation type. The time of disaggregation wasn't influenced by the studied variables $(p<0.05)$. The best homogeneity was verified for the formulation with triple impregnation at $10 \%$.

UNITERMS: Homeopathy. Homeopathic globules/ impregnation. Homeopatic globules/drying.

\section{REFERÊNCIAS BIBLIOGRÁFICAS}

ABFH - Associação Brasileira de Farmacêuticos Homeopatas. Manual de normas técnicas para farmácia homeopática. São Paulo: ABFH, 1992. 24 p. 
ABFH - Associação Brasileira de Farmacêuticos Homeopatas. Manual de normas técnicas para farmácia homeopática. 3. ed. São Paulo: ABFH, 2003. n.p.

ARAÚJO, T.L.; MAZZI, J.L.; CHAUD, M.V.; GUTIERREZ, M.A.; FONTES, O.L. Validação de técnicas e métodos de impregnação de glóbulos homeopáticos. Cult. Homeop., v. 3, n. 9, p. 8-16, 2004.

FARMACOPÉIA Brasileira. 4. ed. São Paulo: Atheneu, 1988. v.1.n.p.

FARMACOPÉIA HOMEOPÁTICA Brasileira. 2. ed. São Paulo: Atheneu, 1997. pt. 1, n.p.

FONTES, O.L. Farmácia Homeopática - Teoria e Prática. 2. ed. Barueri: Manole, 2005. 354 p.

FONTES, O.L.; GUTIERREZ, M.A.; KAZAMA, Y.; MAZZI, J.L. Variação do peso de glóbulos após a impregnação com solução de etanol a 70\%. Informativo $A B F H$, n. 28, p. 6-8, 2002.

GUTIERREZ, M.A. Validação da técnica de impregnação de glóbulos homeopáticos. Rev. Homeop., v. 66, n. 2, p. 27 $35,2001$.

HAHNEMANN, S. Organon da arte de curar. 6. ed. São Paulo: Robe Editorial, 2001. 248 p.
HOWARD, M. A.; NEAU, S. H.; SACK, M. J. PEO and MPEG in high drug load extruded and spheronized beads that are devoid of MCC. Int. J. Pharmac., v. 307, n. 1, p. 66-76, 2006.

LUZ, M.T. A Arte de curar versus a ciência das doenças História social da homeopatia no Brasil. São Paulo: Dynamis, 1996. 342 p.

MONTGOMERY, D.C. Design and analysis of experiments. 5. ed. New York: Wiley \& Sons, 2001. 684 p.

PINHEIRO, M.S. Estudo da uniformidade de dose por conteúdo na impregnação de glóbulos. Rio de Janeiro, 2006. 131p. [Dissertação de Mestrado. Faculdade de Farmácia. Universidade Federal do Rio de Janeiro].

POZETTI, G.L.; SILVA, R.F.P.; PIZZOLITTO, E. L. Técnicas de impregnação de glóbulos homeopáticos: análise laboratorial. Rev. Racine, v. 66, p. 3-8, 2002.

ROCHA, L.M.; AFONSO, C.; LUNA, I.S.; SÁ, I. Otimização da metodologia para impregnação de glóbulos aplicada a farmácias homeopáticas. Homeop. Bras., v. 6, n. 2 , p. $77-81,2000$.

SARTORI, A.V.; DA SILVA, C.E.F.; FERNANDES, C.R.; PONTES, L.M.; BRASIL, L.A.; TEIXEIRA, W.G.; FUTURO, D.O. Avaliação do aspecto e da desagregação de glóbulos impregnados em diferentes métodos e em diferentes proporções. In: CONGRESSO BRASILEIRO DE FARMÁCIA HOMEOPÁTICA, 4., João Pessoa, 2003. Resumos. São Paulo: ABFH, 2003.p. 16.

Recebido para publicação em 25 de julho de 2007 Aceito para publicação em 23 de novembro de 2007 